\title{
Bicoloring Steiner Triple Systems
}

\author{
Charles J. Colbourn \\ Computer Science \\ University of Vermont \\ Burlington VT 05405 \\ colbourn@emba.uvm.edu
}

\author{
Jeffrey H. Dinitz \\ Mathematics and Statistics \\ University of Vermont \\ Burlington VT 05405 \\ Jeff.Dinitz@uvm.edu
}

\author{
Alexander Rosa \\ Mathematics and Statistics \\ McMaster University \\ Hamilton, Ontario, Canada L8S 4K1 \\ rosa@mcmail.cis.mcmaster.ca
}

Submitted: March 31, 1999; Accepted: May 24, 1999.

\begin{abstract}
A Steiner triple system has a bicoloring with $m$ color classes if the points are partitioned into $m$ subsets and the three points in every block are contained in exactly two of the color classes. In this paper we give necessary conditions for the existence of a bicoloring with 3 color classes and give a multiplication theorem for Steiner triple systems with 3 color classes. We also examine bicolorings with more than 3 color classes.

Math Subject Clasification: 05B07
\end{abstract}

\section{Introduction}

Throughout this paper we use notation consistent with that found in [2]. Let $\mathcal{D}=(V, \mathcal{B})$ be a $(v, k, \lambda)$-design. A coloring of $\mathcal{D}$ is a mapping $\varphi: V \rightarrow C$. The elements of $C$ are colors; if $|C|=m$, we have an $m$-coloring of $\mathcal{D}$. For 
each $c \in C$, the set $\varphi^{-1}(c)=\{x: \varphi(x)=c\}$ is a color class. A coloring $\varphi$ of $\mathcal{D}$ is weak (strong) if for all $B \in \mathcal{B},|\varphi(B)|>1(\varphi(B)=k$, respectively), where $\varphi(B)=\cup_{v \in B} \varphi(v)$. Each color class in a weak or strong coloring is an independent set. In a weak coloring, no block is monochromatic (i.e., no block has all its elements the same color), while in a strong coloring, the elements of any block $B$ get $|B|$ distinct colors. The weak [strong] chromatic number of $\mathcal{D}$ is the smallest $m$ for which $\mathcal{D}$ admits a weak [strong] $m$-coloring. Much work has been done on weak and strong colorings; for an extensive survey of these results, the reader is referred to [9].

For triple systems, the following results concerning weak colorings are known.

Theorem 1.1 [3]. For every admissible $v \geq 5$ and any $\lambda$ there exists a weakly 3-chromatic $2-(v, 3, \lambda)$-design.

A modification of Bose's and Skolem's constructions for Steiner triple systems was used to prove:

Theorem 1.2 [1, 4]. A weakly 4-chromatic STS(v) exists for every $v \equiv$ 1 or $3(\bmod 6), v \geq 21$.

In this paper we consider a stronger coloring condition than weak coloring, termed a bicoloring. While a bicoloring is defined for any design, we examine only bicolorings of Steiner triple systems.

A coloring $\varphi$ of $\mathcal{D}$ is a bicoloring if for all $B \in \mathcal{B},|\varphi(B)|=2$, where $\varphi(B)=\cup_{v \in B} \varphi(v)$. This definition implies that in a triple system every triple has two elements in one color class and one in another class, i.e. there are no monochromatic triples nor are there any triples receiving three colors. So, in some sense, a bicoloring of a triple system is an anti-strong weak coloring.

An $m$-bicoloring is a bicoloring with $m$ color classes, and a design admitting an $m$-bicoloring is $m$-bicolorable. A design is $m$-bichromatic if it is $m$-bicolorable but not $(m-1)$-bicolorable.

Example 1.3 A 3-bicolorable STS(13). First, construct an STS(13) by developing the base blocks $\{1,3,9\},\{2,5,6\} \bmod 13$. The color classes are $\{0,1\},\{2,6,8,10,11\},\{3,4,5,7,9,12\}$

In the context of strict colorings of hypergraphs defined recently by Voloshin [12], a bicoloring of an STS is a strict coloring of an STS in which all triples 
are both edges and also co-edges. In [5, 6], Milazzo and Tuza discuss several properties of strict colorings of Steiner triple systems. An easy counting argument [8] establishes that there exist no nontrivial 2-colorable STS (or triple systems of any index $\lambda$ for $v>4$ ), and hence no 2-bichromatic triple systems. In the next section we consider 3-bicolorable (and hence 3-bichromatic) STSs.

While for a weak $m$-chromatic triple system there are some general bounds on the sizes of the color classes (see [9]), in an $m$-bichromatic triple system there is a divisibility condition that the sizes of the color classes must satisfy.

Proposition 1.4 Let $(X, \mathcal{A})$ be an $m$-bicolorable triple system $T S(v, \lambda)$ and assume that the $m$ color classes have sizes $c_{1}, c_{2}, \ldots, c_{m}$. Then

$$
\sum_{i=1}^{m}\left(\begin{array}{l}
c_{i} \\
2
\end{array}\right)=\left(\begin{array}{l}
v \\
2
\end{array}\right) / 3 .
$$

Proof. (See also [6].) Since exactly one of the three pairs of elements covered by any triple of $\mathcal{A}$ has both its elements within one color class, the number of monochromatic pairs must be one third of the total number of pairs.

The divisibility condition is not sufficient. For example, if $c_{i}=c_{j}=2$, and $i \neq j$, there cannot exist an $m$-bicoloring no matter what the size of the other color classes. This is called the duplicity condition.

Since every triple must have two 2-colored pairs, the total number of 2-colored pairs must be even. Hence, we obtain the oddity condition:

Proposition 1.5 Let $(X, \mathcal{A})$ be an $m$-bicolorable triple system $T S(v, \lambda)$ and assume that the $m$ color classes have sizes $c_{1}, c_{2}, \ldots, c_{m}$, then at most one of numbers $c_{1}, c_{2}, \ldots, c_{m}$ can be odd.

If $v$ is an admissible order for STS, every $m$-tuple $\left(c_{1}, c_{2}, \ldots, c_{m}\right)$ (with the $c_{i} \mathrm{~s}$ in nonincreasing order by convention) satisfying the divisibility condition is an $m$-split for $v$.

Every 3-split satisfying the divisibility condition automatically satisfies the oddity condition as well. Indeed, if $(a, b, c)$ is a 3 -split satisfying the divisibility condition for an admissible order $v$, and $a, b, c$ are all odd, then of the four numbers $\left(\begin{array}{l}v \\ 2\end{array}\right),\left(\begin{array}{l}a \\ 2\end{array}\right),\left(\begin{array}{l}b \\ 2\end{array}\right),\left(\begin{array}{l}c \\ 2\end{array}\right)$ either three are even and one is odd, or three are odd and one is even; in either case we have a contradiction.

A similar statement can be shown to be true for 4-splits. However, for $m \geq 5$ there are many $m$-splits satisfying the divisibility condition which 
do not satisfy the oddity condition. Still, these conditions together are not sufficient. In fact, we have the following density condition:

Proposition 1.6 Let $v \equiv 1,3 \quad(\bmod 6)$. If there exists an m-bicolorable $S T S(v)$ with m-split $\left(c_{1}, \ldots, c_{k}, d_{1}, \ldots, d_{m-k}\right)$ (with $\left.0<k<m\right)$, then the inequality

$$
0 \leq\left[\sum_{i=1}^{k}\left(\begin{array}{l}
c_{i} \\
2
\end{array}\right)\right]-\frac{1}{2}\left[\sum_{i=1}^{k-1} \sum_{j=i+1}^{k} c_{i} c_{j}\right] \leq \frac{1}{2}\left[\sum_{i=1}^{k} c_{i}\right] \cdot\left[\sum_{i=1}^{m-k} d_{i}\right]-\ell\left(\sum_{i=1}^{m-k} d_{i}\right)
$$

holds, where

$$
\ell(x)=\left\{\begin{array}{cll}
x / 2 & \text { if } x \equiv 0,2 & (\bmod 6) \\
0 & \text { if } x \equiv 1,3 & (\bmod 6) \\
(x+2) / 2 & \text { if } x \equiv 4 & (\bmod 6) \\
4 & \text { if } x \equiv 5 & (\bmod 6)
\end{array}\right.
$$

Proof. Let $(V, \mathcal{B})$ be a putative $m$-bicolorable $\operatorname{STS}(v)$ with the specified $m$ split. Partition the $m$ color classes into two groups, the first with color class sizes $\left\{c_{1}, \ldots, c_{k}\right\}$ and the second with sizes $\left\{d_{1}, \ldots d_{m-k}\right\}$. We concentrate on triples with one point in the first group and one point in the second (and the location of the third point unknown as yet). A pair with one endpoint in a class in the first group and the other endpoint in a different class of the first group appears in a triple that lies wholly in the first group. Hence we can count triples that lie wholly on the first group, and from this determine the number of triples with two points in a class of the first group and one point in a class of the second group. This count is the left hand side of the inequality given. Each such triple requires two of the pairs between the first and second group. However, some of these pairs may already be required in triples containing two points from a class in the first group and one in a class of the second. The 'correction' $\ell(\ldots)$ is then the minimum number of such triples. If we examine a packing on the points of the second group, say with $x$ points in the second group, we see that $\ell(x)$ is the smallest number of pairs left uncovered by any packing (i.e. the smallest number of edges in the leave graph of any packing). Thus the right hand side of the inequality reflects (one half of) the maximum number of pairs available to form triples with two points in a class of the first group and one point in a class of the second group. 
THE ELECTRONIC JOURNAL OF COMBINATORICS 6 (1999), \#R25

We believe that the necessary conditions given in this section taken together are sufficient for the existence of $m$-bicolorable STSs, at least for $m \leq 5$.

\section{3-bichromatic STS}

\subsection{Necessary conditions}

In order to establish necessary conditions for the existence of a 3-bicolorable STS we first need a number theoretic lemma.

Lemma 2.1 Let $n$ be an integer and $p \geq 5$ a prime factor of $n^{2}+n+1$, then $p \equiv 1 \quad(\bmod 6)$.

Proof. Since $4\left(n^{2}+n+1\right)=(2 n+1)^{2}+3$, if $p$ divides $n^{2}+n+1$, then -3 is a square modulo $p$. Then $1=\left(\frac{-3}{p}\right)$, where $\left(\frac{a}{b}\right)$ is the Legendre symbol. Now $\left(\frac{-3}{p}\right)=\left(\frac{-1}{p}\right)\left(\frac{3}{p}\right)=(-1)^{(p-1) / 2}\left(\frac{p}{3}\right)(-1)^{(p-1)(3-1) / 4}$ by quadratic reciprocity, and hence $1=(-1)^{p-1}\left(\frac{p}{3}\right)$. Since $p$ is odd, $1=\left(\frac{p}{3}\right)$, or equivalently, $p \equiv 1$ $(\bmod 3)$. Again, since $p$ is odd, $p \equiv 1 \quad(\bmod 6)$, the desired conclusion.

We are now in a position to prove the following.

Theorem 2.2 Given an STS(v), and a putative 3-bicoloring with classes of sizes $a, b$, and $v-a-b$. Let $p$ be a prime, $p \equiv 5(\bmod 6)$, and assume $p^{2 i-1}$ divides $v$ for some $i \geq 1$. Then

(a) $p^{i}$ divides $a, b$, and $v-a-b$, and

(b) $p^{2 i}$ divides $v$.

Consequently, if there exists a 3-bicolorable $S T S(v)$, then any prime $p$ dividing $v$ with $p \equiv 5 \quad(\bmod 6)$ must have an even power in the prime factorization of $v$.

Proof. By the divisibility condition,

$$
\left(\begin{array}{l}
a \\
2
\end{array}\right)+\left(\begin{array}{l}
b \\
2
\end{array}\right)+\left(\begin{array}{c}
v-a-b \\
2
\end{array}\right)=\left(\begin{array}{l}
v \\
2
\end{array}\right) / 3
$$


THE ELECTRONiC JOURNAL of COMBinAtorics 6 (1999), \#R25

This simplifies to

$3 a(a-1)+3 b(b-1)+3 v(v-1)-3(2 v-1)(a+b)+3(a+b)^{2}=v(v-1)$.

Hence

$$
3 a^{2}+3 b^{2}+3 a b+v(v-1)-3 v(a+b)=0 .
$$

Now suppose that $v$ is a multiple of $p^{2 i-1}$, and treat this equation modulo $p^{2 i-1}$ to get that $3 a^{2}+3 b^{2}+3 a b \equiv 0 \quad\left(\bmod p^{2 i-1}\right)$. Since 3 and $p$ are relatively prime,

$$
a^{2}+b^{2}+a b \equiv 0 \quad\left(\bmod p^{2 i-1}\right) .
$$

Now one obvious solution has $a \equiv b \equiv 0 \quad\left(\bmod p^{i}\right)$. If this is not the solution, then without loss of generality say $a$ is not a multiple of $p^{i}$. We will obtain a contradiction in this case.

Let $k$ be such that $p^{k}$ divides $a$, but $p^{k+1}$ does not divide $a$, with $0 \leq k \leq$ $i-1$. From $(3), p^{2 k}$ divides $b^{2}+a b=b(b+a)$. Since $p^{k}$ divides $a$, it follows that $p^{k}$ also must divide $b$. Let $\hat{a}=a / p^{k}$ and $\hat{b}=b / p^{k}$. Dividing (3) by $p^{2 k}$ yields the equation $\hat{a}^{2}+\hat{b}^{2}+\hat{a} \hat{b} \equiv 0 \quad\left(\bmod p^{2 i-2 k-1}\right)$. Since $p$ does not divide $\hat{a}$ we can multiply by $\hat{a}^{-1}$ modulo $p^{2 i-2 k-1}$ to obtain

$$
1+n^{2}+n \equiv 0 \quad\left(\bmod p^{2 i-2 k-1}\right)
$$

where $n$ is $\hat{b} \hat{a}^{-1} \quad(\bmod p)$. But now by Lemma 2.1, this does not happen when $p \equiv 5 \quad(\bmod 6)$. Thus, $p^{i}$ divides $a, b$ and hence also $v-a-b$.

Now look at (2) again. Since $p^{2 i}$ is a divisor of the terms $3 a^{2}, 3 b^{2}, 3 a b$ and $3 v(a+b)$, we find that $p^{2 i}$ must divide $v(v-1)$. Since $p$ divides $v$, then $p$ and $v-1$ are relatively prime, and hence $p^{2 i}$ must divide $v$. This completes the proof.

We conjecture that the necessary condition given in Theorem 2.2 is also sufficient for the existence of 3-bichromatic STS, and in the next section we find 3-bicolorable $\operatorname{STS}(v)$ s for many orders of $v$.

\section{$2.2 \quad$ Existence}

We begin this section with a multiplication theorem. One key ingredient of the construction is a special type of latin square. We first prove a theorem about these latin squares. 
THE ELECTRONIC JOURNAL OF COMBINATORICS 6 (1999), \#R25

Write $n=a+b+c$. Let $A, B$ and $C$ be disjoint sets of sizes $a, b$ and $c$, respectively. A latin square with rows, columns, and symbols indexed by $A \cup B \cup C$ is called $(a, b, c)$-forbidden if in cell $(r, g)$ we find symbol $s$ satisfying:

$r$ in $A$ and $g$ in $A$ implies $s$ not in $C$

$r$ in $A$ and $g$ in $B$ implies $s$ not in $B$

$r$ in $A$ and $g$ in $C$ implies $s$ not in $A$

$r$ in $B$ and $g$ in $A$ implies $s$ not in $B$

$r$ in $B$ and $g$ in $B$ implies $s$ not in $A$

$r$ in $B$ and $g$ in $C$ implies $s$ not in $C$

$r$ in $C$ and $g$ in $A$ implies $s$ not in $A$

$r$ in $C$ and $g$ in $B$ implies $s$ not in $C$

$r$ in $C$ and $g$ in $C$ implies $s$ not in $B$

Now $(a, b, c)-,(b, c, a)$ - and $(c, a, b)$-forbidden latin squares are the same. Similarly, $(b, a, c)-,(a, c, b)-$ and $(c, b, a)$-forbidden latin squares are the same.

Lemma 2.3 An $(a, b, c)$-forbidden latin square of order $n$ exists if and only if $\max (a, b, c) \leq n / 2$.

Proof. Necessity is obvious. To prove sufficiency, we assume without loss of generality that $c=\max (a, b, c)$, so that we need only treat cases when $a \leq b \leq c$, and when $b<a \leq c$. Let $X$ be a set of $(a+b-c)$ symbols disjoint from $A, B$ and $C$. We are going to form a partial latin square $P$. The construction differs slightly in the two cases.

If $a \leq b$ form an $(a+b) \times(a+b)$ latin square $R$ with rows and columns indexed by $A \cup B$, and symbols indexed by $C \cup X$, so that $R$ contains an $a \times a$ subsquare on the rows and columns indexed by $A$, and the symbols in the subsquare containing all symbols in $X$ (and possibly some of the symbols in $C$ ). From $R$, form a partial latin square $P$ as follows. Remove the $a \times a$ subsquare and place an $a \times a$ subsquare on the symbols in $A$ in its place.

When $a>b$, instead first place an $a \times a$ square on symbols in $A$ on the subsquare with rows and columns indexed by $A$; a $b \times a$ latin rectangle on a subset $C_{a}$ of a symbols in $C$ on the rectangle with rows indexed by $B$ and columns by $A$; an $a \times b$ latin rectangle on the subset $C_{a}$ of symbols on the rectangle with rows indexed by $A$ and columns by $B$. Then fill the $b \times b$ 
subsquare with rows and columns indexed by $B$ using a latin square on the symbols $\left(C-C_{a}\right) \cup X$ (this is indeed $b$ symbols as required).

Now the two cases merge. Delete all occurrences of elements in $X$ from the square; all appear in the subarray with rows and columns indexed by $B$. Form a bipartite graph, with one class being the rows indexed by $B$, the other being the columns indexed by $B$, with a row and column vertex made adjacent whenever the symbol was one of those in $X$. Color this bipartite graph in $b$ colors, so that the coloring is equalized (proper and every color appearing the same number of times as every other, i.e. $a+b-c$ times). This can be done by a theorem of de Werra [13]. Now whenever an edge of this bipartite graph gets the $i$ th color, place in the corresponding cell the $i$ th symbol in $B$. At this point, $P$ is a partial latin square with symbols from $A, B$ and $C$. In fact, every symbol in $A$ appears $a$ times, every one in $B$ occurs $a+b-c$ times, and every one in $C$ is at least $b$ times. It follows from Ryser's Theorem [10] that $P$ can be completed to a latin square, since $n=a+b+c$ and so every symbol occurs at least $(a+b)+(a+b)-(a+b+c)=a+b-c$ times as required.

That this forces the $(a, b, c)$-forbidden requirements (taking of course all rows and columns added in the embedding to be those indexed by $C$ ) is easy counting. To help check it, here are the counts on numbers of symbols from each of $A, B$ and $C$ in each of the subarrays:

Symbols from $A$ :
\begin{tabular}{l|ccc|}
$A$ & $B$ & $C$ \\
\cline { 2 - 4 }$A$ & $a^{2}$ & 0 & 0 \\
$B$ & 0 & 0 & $a b$ \\
$C$ & 0 & $a b$ & $a(c-b)$ \\
\cline { 2 - 4 } & &
\end{tabular}

\begin{tabular}{c|ccc|}
\multicolumn{4}{c}{ Symbols from $B:$} \\
\multicolumn{1}{c}{$A$} & $B$ & $C$ \\
\cline { 2 - 4 }$A$ & 0 & 0 & $a b$ \\
$B$ & 0 & $b(a+b-c)$ & $b(c-a)$ \\
$C$ & $a b$ & $b(c-a)$ & 0 \\
\cline { 2 - 4 } & &
\end{tabular}

Symbols from $C$ :

\begin{tabular}{c|ccc|}
\multicolumn{1}{c}{$A$} & $B$ & $C$ \\
\cline { 2 - 4 }$A$ & 0 & $a b$ & $a(c-b)$ \\
$B$ & $a b$ & $b(c-a)$ & 0 \\
$C$ & $a(c-b)$ & 0 & $c^{2}-a(c-b)$ \\
\cline { 2 - 4 } & &
\end{tabular}

This finishes the existence proof for $(a, b, c)$-forbidden latin squares.

Next we make a simple observation, that in an $(a, b, c)$-bicolored STS, the size $c$ satisfies $c \leq(a+b)$ unless $(a, b, c)=(0,1,2)$ (and $v=3)$ or $(a, b, c)=$ $(1,2,4)$ (and $v=7)$. The proof is easy: By the divisibility condition, $\left(\begin{array}{l}a \\ 2\end{array}\right)+$ 
$\left(\begin{array}{l}b \\ 2\end{array}\right)+\left(\begin{array}{l}c \\ 2\end{array}\right)$ must equal $\left(\begin{array}{c}a+b+c \\ 2\end{array}\right) / 3$. Simplify to get $a^{2}+b^{2}+c^{2}-(a+b+c)-(a b+$ $a c+b c)=0$. Now assume that $c>a+b$. The left side of this expression is minimized when $a=b$, but then one can check that for this side to be less than or equal to 0 , one must have $v \leq 16$. Now these remaining orders can be checked by hand.

This underlies the one exceptional case in the direct product to follow.

Theorem 2.4 If there exists an $(a, b, c)$-bicolorable $S T S(u)$ with $c=\max (a, b, c)$ and $c \leq a+b$, and if there exists an $(x, y, z)$-bicolorable STS $(v)$, then there exists an $(a x+b y+c z, a y+b z+c x, a z+b x+c y)$-bicolorable STS $(u v)$.

Proof. Define sets $V_{i j}$ of elements with $0 \leq i<v$ and $j \in\{0,1,2\}$, so that $V_{i j}$ has $a, b, c$ elements for $j=0,1,2$ respectively, when $0 \leq i<x ; b, c, a$ elements for $j=0,1,2$ respectively, when $x \leq i<x+y$; and $c, a, b$ elements for $j=0,1,2$ respectively, when $x+y \leq i<v$. The union of $V_{i j}$ for $0 \leq i<v$ then has $a x+b y+c z, b x+c y+a z$, and $c x+a y+b z$ elements for $j=0,1,2$, respectively.

For $i=0, \ldots, v-1$, place on the union of $V_{i j}$ for $j=0,1,2$ an $(a, b, c)-$ bicolored STS $(u)$ in which the color classes are $V_{i 0}, V_{i 1}$ and $V_{i 2}$. Now choose an $(x, y, z)$-bicolored $\operatorname{STS}(v)$ in which the color classes are $\{0, \ldots, x-1\},\{x, \ldots, x+$ $y-1\},\{x+y, \ldots, v-1\}$, and let $\mathcal{B}$ be its blocks. Call the colors in this coloring 0,1 and 2. Whenever $\{f, g, h\} \in \mathcal{B}$, suppose without loss of generality that $f$ and $g$ have the same color $k$ in the bicoloring of the $\operatorname{STS}(v)$. When $h$ has color $(k+2) \bmod 3$, form an $(a, b, c)$-forbidden latin square; when $h$ has color $(k+1) \bmod 3$, instead form a $(b, a, c)$-forbidden latin square. Use the latin square to construct triples in the obvious way (i.e., form the transversal design $\operatorname{TD}(3, u)$ from the latin square and align the row, column, and symbol classes of sizes $a, b, c$ on the corresponding $V_{f j}^{\prime} s, V_{g j}^{\prime} s$ and $\left.V_{h j}^{\prime} s\right)$.

The result is a bicolorable $\operatorname{STS}(u v)$ whose color classes have the specified sizes.

Corollary 2.5 If there exists a 3-bicolorable STS(u) and a 3-bicolorable STS(v), then there exists a 3-bicolorable STS(uv).

Proof. The corollary follows from Theorem 2.4 except possibly when $u$ and $v$ both are 3 or 7 . Examples of 3-bicolorable $\operatorname{STS}(9), \operatorname{STS}(21)$, and $\operatorname{STS}(49)$ are easily found. 
THE ELECTRONIC JOURNAL OF COMBINATORICS 6 (1999), \#R25

If there exists a 3-bicolorable $\operatorname{STS}(v)$ with a 3 -split $(a, b, c)$, then $(2)$ must be satisfied. Using $(2)$ for $v \equiv 1,3 \quad(\bmod 6), v \leq 97$ yields the following solutions.

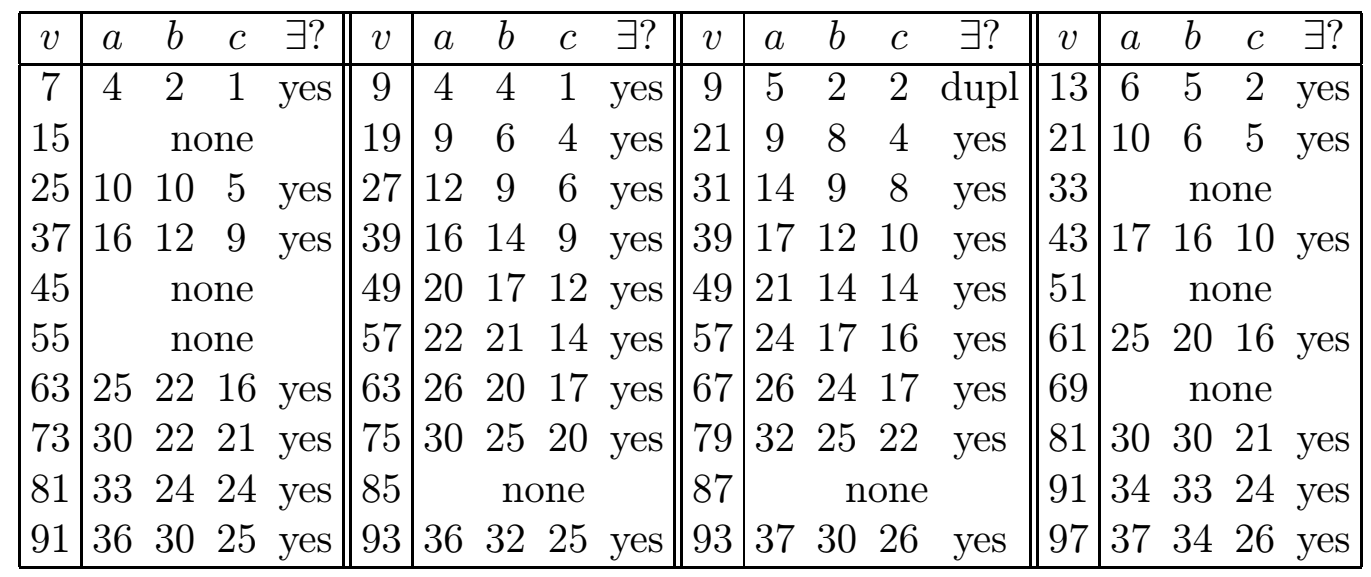

We have obtained solutions for all of these meeting the duplicity condition, using Stinson's hill-climbing algorithm for triple systems [11] (or see [2], p. 730) modified so that only 2-colored triples could be constructed. We have also constructed a 3 -bicolorable $\operatorname{STS}(v)$ s for all $v \equiv 1,3(\bmod 6)$, $99 \leq v<1000$, satisfying the condition given in Theorem 2.2 and every possible 3 -split satisfying the divisibility condition.

Theorem 2.6 For every $v \equiv 1,3 \quad(\bmod 6), v<1000$ satisfying the necessary condition given in Theorem 2.2 and for all 3-splits $(a, b, c)$ satisfying the divisibility condition, there exists a 3-bicolorable STS $(v)$ with color classes of sizes $a, b$, and $c$.

Conjecture 2.7 For every $v \equiv 1,3 \quad(\bmod 6)$, satisfying the condition in Theorem 2.2 and for all 3-splits $(a, b, c)$ for $v$ satisfying the divisibility and duplicity conditions, there exists a 3-bicolorable STS $(v)$ with color classes of sizes $a, b$, and $c$.

¿From Theorem 2.6 and Corollary 2.5, we have our main result on the existence of 3-bicolorable STSs:

Theorem 2.8 Let $v \equiv 1,3 \quad(\bmod 6)$ and assume that in the prime factorization of $v$ no prime congruent to $5(\bmod 6)$ appears with an odd exponent. Further assume that all prime factors $p$ congruent to 1 (mod 6) are less than 1000 and that all prime factors $p$ congruent to $5(\bmod 6)$ satisfy $p^{2}<1000$, then there exists a 3-bicolorable STS $(v)$. 
THE ELECTRONIC JOURNAL OF COMBINATORICS 6 (1999), \#R25

\section{4- and 5-bicolorable STS}

The following has been observed, in effect, in [6].

Theorem 3.1 If there exists an $m$-bicolorable $S T S(v)$ with $m$-split $\left(c_{1}, \ldots, c_{m}\right)$, then there exists an $(m+1)$-bicolorable $S T S(2 v+1)$ with an $(m+1)$-split $\left(v+1, c_{1}, \ldots, c_{m}\right)$.

Proof. Apply the well-known 2v+1 construction (cf. [3], Chapter 3), and color all elements not in the $\operatorname{sub}-\operatorname{STS}(v)$ with a new color.

A variant of this gives a different $(m+1)$-split:

Theorem 3.2 If there exists an m-bicolorable STS $(v)$ with $m$-split $\left(c_{1}, \ldots, c_{m}\right)$, then there exists an $(m+1)$-bicolorable $S T S(2 v+1)$ with an $(m+1)$-split $\left(2 c_{1}, 2 c_{2}, \ldots, 2 c_{m}, 1\right)$.

Proof. Let $(V, \mathcal{B})$ be the $m$-bicolorable $\operatorname{STS}(v)$. We form an $\operatorname{STS}(2 v+1)$ on $(V \times\{0,1\}) \cup\{\infty\}$ as follows. Whenever $\{x, y, z\} \in \mathcal{B}$, form the blocks $\left\{x_{i}, y_{j}, z_{k}\right\}$ for $(i, j, k)=(0,0,0),(0,1,1),(1,0,1)$, and $(1,1,0)$. Then form the blocks $\left\{\infty, x_{0}, x_{1}\right\}$ for $x \in X$. Color $x_{i}$ for $i \in\{0,1\}$ using the same color as was assigned to $x$ in the $\operatorname{STS}(v)$. Then assign $\infty$ a new color.

That there exist $m$-bicolorable STSs with arbitrarily large $m$ is now immediate. However, in this section we restrict our attention to the case of 4- and 5-bicolorings as these display some marked contrasts to the case of 3-bicolorings. For example, while Theorem 2.2 ensures that there exist infinitely many admissible orders $v$ for which no 3-bicolorable STS can exist, this does not appear to be so in the case of 4- or 5-bicolorings.

Another somewhat less obvious recursion is proved:

Theorem 3.3 If there exists a m-bicolorable $S T S(v)$ with $m$-split $\left(c_{1}, \ldots, c_{m}\right)$, then there exists a $(m+2)$-bicolorable $S T S(5 v+4)$ with $(m+2)$-split $(2 v+$ $\left.2,2 v+2, c_{1}, \ldots, c_{m}\right)$.

Proof. Let $V=\left\{a_{j}: j=1, \ldots, v\right\}$, and suppose $(V, \mathcal{B})$ is the $m$-colorable $\operatorname{STS}(v)$. We construct a $(m+2)$-bicolorable $\operatorname{STS}(5 v+4)$ on the $(5 v+4)$-set $W=Z_{2 v+2} \times\{1,2\} \cup V$ as follows.

Consider the graph $G$ with the vertex-set $Z_{2 v+2}$ and the edge-set $\{\{x, y\}$ : $|x-y| \in\{(v+3) / 2,(v+5) / 2, \ldots, v+1\} ; G$ is regular of degree $v$, and thus, 
by the Stern-Lenz Lemma (cf. [3], Chapter 1), $G$ has a 1-factorization, say $\left\{F_{1}, \ldots, F_{v}\right\}$. Let further $S=\left\{\left(a_{r}, b_{r}\right): r=1,2, \ldots,(v+1) / 2\right\}, b_{r}-a_{r}=r$ be a Skolem sequence of order $(v+1) / 2$ if $(v+1) / 2 \equiv 0,1(\bmod 4)$, or a hooked Skolem sequence of order $(v+1) / 2$ if $(v+1) / 2 \equiv 2,3 \quad(\bmod 4)(\mathrm{cf}$. [3], Chapter 2).

Define now the following sets of triples (for the sake of brevity we write $x_{i}$ for $(x, i)$ etc. $)$.

$\left.\mathcal{P}=\left\{a_{j}, x, y\right\}:\{x, y\} \in F_{j}^{i}, i=1,2, j=1,2, \ldots, v\right\}$,

$\mathcal{Q}=\left\{\left\{i_{2},(i+r-1)_{1},\left(i+a_{r}-1\right)_{1}\right\}: r=1, \ldots,(v+1) / 2, i \in Z_{2 v+2}\right\}$,

$\mathcal{R}=\left\{\left\{i_{1},(i+2 v+1-r)_{2},\left(i+2 v+1-a_{r}\right)_{2}\right\}: r=1, \ldots,(v+1) / 2, i \in\right.$ $\left.Z_{2 v+2}\right\}$.

Then $(W, \mathcal{B} \cup \mathcal{P} \cup \mathcal{Q} \cup \mathcal{R})$ is an $\operatorname{STS}(5 v+4)$. Moreover, this STS is $(m+2)$-bicolorable with color classes $Z_{2 v+2} \times\{1\}, Z_{2 v+2} \times\{2\}$, and the $m$ color classes of the original $m$-bicoloring of $(V, \mathcal{B})$.

A specific recursion for special 4-splits can also be established:

Theorem 3.4 Suppose that there exists a 4-bicolorable STS(v) with 4-split $(x, y, z, 1)$ and a 4-bicolorable STS $(u)$ with 4 -split $(a, b, c, 1)$, so that $c=$ $\max (a, b, c)$ and $c \leq a+b$. Then there exists a 4-bicolorable $\operatorname{STS}\left(\frac{1}{2}(u-\right.$ $1)(v-1)+1)$ with 4 -split $\left(\frac{1}{2}(a x+b y+c z), \frac{1}{2}(a y+b z+c x), \frac{1}{2}(a z+b x+c y), 1\right)$.

Proof. Define sets $V_{i j}$ of elements with $0 \leq i<\frac{v-1}{2}$ and $j \in\{0,1,2\}$, so that $V_{i j}$ has $a, b, c$ elements for $j=0,1,2$ respectively, when $0 \leq i<\frac{x}{2}$; $b, c, a$ elements for $j=0,1,2$ respectively, when $\frac{x}{2} \leq i<\frac{x+y}{2}$; and $c, a, b$ elements for $j=0,1,2$ respectively, when $\frac{x+y}{2} \leq i<\frac{v-1}{2}$. The union of $V_{i j}$ for $0 \leq i<\frac{v-1}{2}$ then has $\frac{1}{2}(a x+b y+c z), \frac{1}{2}(a y+b z+c x), \frac{1}{2}(a z+b x+c y)$ elements for $j=0,1,2$, respectively. Add a new point $\infty$.

For $i=0, \ldots, \frac{v-1}{2}-1$, place on the union of $V_{i j}$ for $j=0,1,2$ together with $\infty$, an $(a, b, c, 1)$-bicolored $\operatorname{STS}(u)$ in which the color classes are $V_{i 0}, V_{i 1}$, $V_{i 2}$, and $\{\infty\}$. Next consider the 4 -bicolored $\operatorname{STS}(v)$ with color classes $\{i, \bar{i}$ : $\left.0 \leq i<\frac{x}{2}\right\},\left\{i, \bar{i}: \frac{x}{2} \leq i<\frac{x+y}{2}\right\},\left\{i, \bar{i}: \frac{x+y}{2} \leq i<\frac{v-2}{2}\right\}$, and $\{\infty\}$. Call the colors in this coloring $0,1,2$, and 3 , where $\infty$ gets color 3. Partition each set $V_{i j}$ into two sets $X_{i j}$ and $\bar{X}_{i j}$ of equal size, for each $0 \leq i<\frac{v-1}{2}$ and $j \in\{0,1,2\}$. Consider a block of the $\operatorname{STS}(v)$ not containing $\infty$, say $\{f, g, h\}$. For each element $t \in\{f, g, h\}$, choose one of $X_{i j}$ or $\bar{X}_{i j}$ depending upon whether $t$ is of the form $i$ or $\bar{i}$. On the three corresponding sets, latin squares of side $\frac{u-1}{2}$ are used to form triples as in Theorem 2.4. 
THE ELECTRONIC JOURNAL OF COMBINATORICS 6 (1999), \#R25

Table 1 lists all possible 4-splits for $v \leq 99$, and Table 2 all possible 5splits for $v \leq 87$ meeting the divisibility conditions. The entry 'yes' indicates that the corresponding STS has been found by hill climbing or by the constructions given in this paper. The entries 'dupl', 'odd', and 'den' indicate that the parameters fail the duplicity, oddity, or density condition, respectively. As in the case of 3-bicoloring, we have actually generated all possible 4-splits satisfying the divisibility and duplicity conditions for all $v \leq 157$, and all possible 5-splits satisfying the divisibility, duplicity, oddity, and density conditions for $v \leq 105$. We then used the hill-climbing algorithm to verify that in each case there exists a bicolorable STS with the corresponding split.

In view of the above, the following conjectures appear reasonable.

Conjecture 3.5 A 4-bicolorable $S T S(v)$ exists if and only if $v \equiv 1,3(\bmod 6)$ $, v \geq 15, v \neq 21,31$.

Conjecture 3.6 A 5-bicolorable $S T S(v)$ exists if and only if $v \equiv 1,3(\bmod 6)$, $v \geq 31, v \neq 33,37,43,45,61$.

\section{Conclusion}

If an $\operatorname{STS}(v)$ is $m$-bicolorable then $m \leq\left\lceil\log _{2}(v+1)\right\rceil[5,6]$. Moreover, for all $v=2^{n}-1$ there exists an $\operatorname{STS}(v)$ for which this bound is attained. The corresponding STS is the projective $\operatorname{STS}(v)$ (see, e.g., [3]).

If we define the spectrum $\mathcal{C}(v)$ for bicolorings by $\mathcal{C}(v)=\{m$ : there exists an $m$-bicolorable STS $(v)\}$ then we have $\mathcal{C}(7)=\mathcal{C}(9)=\mathcal{C}(13)=\{3\}, \mathcal{C}(15)=$ $\{4\}, \mathcal{C}(19)=\{3,4\}, \mathcal{C}(21)=\{3\}, \mathcal{C}(25)=\mathcal{C}(27)=\{3,4\}, \mathcal{C}(31)=\{3,5\}$, $\mathcal{C}(33)=\{4\}$.

Thus $\mathcal{C}(v)$ is not necessarily an interval. Can one characterize those orders $v$ for which $\mathcal{C}(v)$ is an interval? It is conceivable that 31 is the only admissible order $v$ for which $\mathcal{C}(v)$ is not an interval.

\section{Acknowledgments}

We thank Vitali Voloshin for suggesting the problem, and Dave Dummit for assistance with the proof of Lemma 2.1. Research of the authors is supported by ARO grant DAAG55-98-1-0272 (Colbourn), and NSERC Canada grant OGP007268 (Rosa). 


\begin{tabular}{|c|c|c|c|c|c|c|c|c|c|c|c|c|c|c|c|c|}
\hline & $a$ & $b$ & $c$ & $d$ & & & $a$ & & & & & $v$ & $a$ & $C_{1}$ & & \\
\hline 15 & 8 & 4 & 2 & 1 & & & 10 & 5 & 2 & 2 & dunl & 19 & 10 & 4 & 1 & \\
\hline & 8 & 8 & 2 & 1 & & & & & & & & 25 & 12 & 4 & 1 & te \\
\hline & 4 & 6 & 5 & 2 & & & 12 & 10 & 4 & 1 & & & \multicolumn{4}{|c|}{ none } \\
\hline & 16 & 10 & 5 & 2 & & & 16 & 8 & 8 & & vos & 33 & 1413 & 34 & 2 & \\
\hline & & 14 & 5 & 2 & & & 20 & 9 & 6 & & & & 813 & 6 & & \\
\hline & 8 & 12 & 8 & 1 & & 3 & 22 & 10 & 6 & & & 3 & 22 & 8 & $I$ & \\
\hline & 21 & 10 & 10 & 2 & & 43 & 20 & 12 & 10 & 1 & & 43 & $18 \quad 17$ & 4 & 4 & \\
\hline & & 16 & 8 & 1 & & 45 & 22 & 13 & 6 & & & 45 & 2114 & 8 & 2 & \\
\hline & 4 & 14 & 6 & 5 & & 9 & 1 & 18 & 8 & & & & 2610 & 10 & 5 & \\
\hline & 5 & 14 & 8 & 4 & & & 20 & 20 & 10 & 1 & & & 12 & 9 & 6 & \\
\hline & 6 & 17 & 8 & 4 & & 55 & 24 & 18 & 12 & 1 & & 57 & 2422 & 6 & 5 & \\
\hline & & 21 & 10 & 2 & & 7 & & 0 & 2 & & & & 016 & 10 & 5 & \\
\hline & 30 & 14 & 13 & 4 & & 61 & 29 & 18 & 10 & 4 & & 1 & 2820 & 9 & 4 & \\
\hline & 8 & 18 & 13 & 2 & & 61 & 25 & 24 & 8 & 4 & & 3 & 3214 & 9 & 8 & \\
\hline & & 18 & 14 & 2 & & & & 2 & 9 & & & & 818 & 16 & 1 & \\
\hline & 33 & 18 & 8 & 8 & & 67 & 32 & 20 & 9 & 6 & & 7 & 3021 & 14 & 2 & \\
\hline & & 16 & 14 & 5 & & & 32 & 22 & 10 & 5 & & & 2926 & 10 & 4 & \\
\hline & 8 & 26 & 13 & 2 & & & 36 & 18 & 13 & 6 & & 3 & 3422 & 213 & 4 & \\
\hline & 33 & 24 & 12 & 4 & $\mathrm{y}$ & 73 & 32 & 26 & 10 & 5 & S & 73 & 3220 & 20 & 1 & \\
\hline & & 28 & & 2 & & & & 28 & 16 & & & 5 & 316 & 12 & 9 & \\
\hline & 37 & 18 & 14 & 6 & & 75 & 36 & 22 & 9 & 8 & & 5 & $\begin{array}{ll}36 & 18\end{array}$ & $8 \quad 17$ & 4 & \\
\hline & 4 & 21 & 18 & 2 & & 75 & 33 & 26 & 12 & 4 & yes & 5 & 3324 & 416 & 2 & \\
\hline & & 28 & 9 & 6 & & & 3 & 24 & 8 & 1 & & & 030 & $\begin{array}{ll}0 & 10\end{array}$ & 5 & \\
\hline & 10 & 17 & 12 & 10 & $\mathrm{y}$ & 79 & 40 & 16 & 14 & 9 & & 79 & 3822 & 213 & 6 & \\
\hline & 6 & 25 & 4 & 4 & $\mathrm{yc}$ & & 34 & 99 & 10 & 6 & & 9 & 3424 & 20 & 1 & \\
\hline & & 28 & 16 & 2 & & & 32 & 28 & 18 & 1 & & 1 & 4021 & 10 & 10 & \\
\hline & 38 & 24 & 14 & 5 & yes & 81 & 34 & 30 & 13 & 4 & ves & 81 & 3332 & 8 & 8 & \\
\hline & & 21 & 14 & 8 & & & 41 & 24 & 12 & 8 & & & $38 \quad 29$ & 912 & 6 & \\
\hline & 38 & 24 & 21 & 2 & & & 36 & 32 & 9 & 8 & & & $36 \quad 29$ & 918 & 2 & \\
\hline & 44 & 17 & 16 & 10 & $\mathrm{yc}$ & 87 & 41 & 22 & 20 & 4 & yes & 87 & $40 \quad 26$ & 617 & 4 & \\
\hline & & 3 & 20 & 1 & & & 44 & 25 & 14 & 8 & & & 3834 & 414 & 5 & \\
\hline 9 & 46 & 21 & 18 & 8 & & 93 & 45 & 26 & 12 & 10 & yes & 93 & $45 \quad 22$ & 220 & 6 & \\
\hline & & 28 & 12 & 9 & & & 4 & 30 & 16 & 5 & & & $42 \quad 29$ & 918 & 4 & \\
\hline & & 30 & 21 & 2 & & & 38 & 36 & 13 & 6 & & 97 & $46 \quad 29$ & 912 & 10 & \\
\hline 9 & & 24 & 22 & 5 & & 97 & 45 & 26 & 22 & 4 & & 97 & 4234 & 416 & 5 & \\
\hline$g$ & & 3 & 14 & 6 & & 97 & 40 & 36 & 17 & 4 & & 97 & 4034 & 421 & 2 & \\
\hline 9 & & 32 & 24 & 1 & & 99 & 50 & 21 & 14 & 14 & ye & 99 & $50 \quad 20$ & 017 & 12 & $\mathrm{yc}$ \\
\hline 9 & & 2 & 16 & 10 & & 99 & 48 & 26 & 17 & 8 & & 99 & $46 \quad 25$ & 524 & 4 & \\
\hline & & & 10 & 10 & $\mathrm{y}$ & 99 & 42 & 28 & 28 & 1 & $\mathrm{yt}$ & 99 & 4138 & $8 \quad 12$ & 8 & \\
\hline & 40 & 34 & 24 & 1 & & 99 & & 38 & 21 & & & & & & & \\
\hline
\end{tabular}

Table 1: 4-splits 


\begin{tabular}{|c|c|c|c|c|c|c|c|c|c|c|c|c|c|c|c|c|c|c|c|}
\hline$v$ & $a$ & & $c$ & $d$ & & $\exists ?$ & $v$ & $a$ & $b$ & $c$ & $d$ & $\exists ?$ & & $a$ & & $c$ & $d$ & & \\
\hline 31 & 16 & 8 & 4 & 列 & & & 33 & & & nor & & & 37 & \multicolumn{6}{|c|}{ none } \\
\hline & 20 & 10 & 5 & 2 & & dupl & 9 & 20 & 10 & 4 & 4 & 1 yes & 39 & 20 & 8 & 8 & 2 & 1 & yes \\
\hline & 19 & 11 & 7 & 1 & & odd & 9 & 17 & 15 & 3 & 3 & 1 odd & 39 & 16 & 16 & 4 & 2 & & \\
\hline & 21 & 13 & 5 & 3 & & odd & 3 & 21 & 11 & 9 & 1 & 1 odd & 45 & \multicolumn{6}{|c|}{ none } \\
\hline & 20 & 20 & 5 & 2 & 2 & dupl & 49 & 20 & 20 & 4 & 4 & 1 yes & 51 & 26 & 13 & 6 & 4 & 2 & den \\
\hline & 26 & 12 & 8 & 4 & & & 1 & 25 & 15 & 5 & 51 & 1 odd & 51 & 24 & 17 & 4 & 4 & & den \\
\hline & 24 & 16 & 8 & 2 & & yes & 1 & 23 & 17 & 9 & 1 & 1 odd & 5 & 28 & 4 & 6 & 5 & & yes \\
\hline & 28 & 12 & 10 & 4 & 1 & & 5 & 27 & 15 & 9 & 3 & 1 odd & 5 & 26 & 18 & 5 & 4 & 2 & den \\
\hline & 25 & 19 & 7 & 3 & & odd & 5 & 24 & 20 & 8 & 21 & 1 yes & 57 & 28 & 17 & 4 & 4 & & den \\
\hline & 28 & 6 & 8 & 4 & & ves & 7 & 26 & 20 & 5 & 42 & 2 den & 1 & 30 & 18 & 5 & 4 & & den \\
\hline & 28 & 21 & 6 & 4 & 2 & den & 63 & 31 & 15 & 13 & 3 & 1 odd & 63 & 30 & 20 & 6 & 5 & 2 & yes \\
\hline & & & 8 & 4 & & den & & & 16 & 10 & 5 & 2 yes & 7 & & 16 & 8 & 8 & 1 & yes \\
\hline & 34 & 14 & 13 & 4 & 2 & es & 67 & 32 & 21 & 6 & 6 & 2 den & 67 & 32 & 20 & 10 & 4 & 1 & yes \\
\hline & 32 & 16 & 16 & 2 & 1 & yes & 67 & 31 & 17 & 17 & 1 & 1 odd & 67 & 30 & 24 & 6 & 5 & 2 & yes \\
\hline & 29 & 5 & 7 & 5 & & odd & & 9 & 23 & 13 & 1 & 1 odd & 7 & & 26 & 0 & 4 & & \\
\hline & 34 & 20 & 6 & 5 & 4 & den & 69 & 30 & 26 & 5 & 4 & 4 den & 69 & 30 & 25 & 10 & 2 & 2 & dupl \\
\hline & 28 & 28 & 6 & 5 & 2 & yes & 3 & 30 & 21 & 6 & 6 & 4 den & 73 & 36 & 20 & 10 & 5 & 2 & yes \\
\hline & 36 & 20 & 8 & 8 & & & & & 16 & 16 & 4 & $1 \mathrm{yc}$ & 3 & & 24 & 8 & 5 & & $\theta$ \\
\hline 7 & 30 & 29 & 8 & 4 & 2 & yes & 75 & 38 & 16 & 14 & 5 & 2 yes & 75 & 37 & 21 & 9 & 5 & 3 & odd \\
\hline & 37 & 19 & 13 & 5 & 1 & odd & 5 & & 25 & 5 & $5:$ & 5 odd & 5 & 4 & 26 & 8 & 5 & 2 & yes \\
\hline & 33 & 27 & 9 & 5 & & odd & & 3 & 28 & 10 & 4 & 1 yes & 79 & 40 & 20 & 9 & 6 & 4 & is \\
\hline 7 ? & 40 & 18 & 13 & 6 & 2 & yes & 79 & 40 & 18 & 12 & 8 & 1 yes & 79 & 39 & 21 & 11 & 7 & 1 & odd \\
\hline & 3 & & & 5 & & & & & 24 & 9 & 4 & 4 yes & & 37 & 26 & 6 & 6 & & den \\
\hline 7 & 37 & 25 & 11 & 3 & 3 & odd & 79 & 37 & 23 & 15 & 3 & 1 odd & 79 & 36 & 26 & 12 & 4 & 1 & ves \\
\hline 7 & 36 & 24 & 16 & 2 & 1 & yes & 79 & 35 & 27 & 13 & 3 & 1 odd & 79 & 34 & 30 & 6 & 5 & 4 & den \\
\hline & 33 & 31 & 7 & 5 & & odd & 8 & 3 & 26 & 9 & 6 & 2 yes & & 37 & 28 & 6 & 6 & & der \\
\hline 8 & 36 & 29 & 8 & 6 & 2 & den & 81 & 36 & 28 & 12 & 4 & 1 yes & 85 & 42 & 24 & 8 & 6 & 5 & den \\
\hline & 41 & 26 & 6 & 6 & 6 & den & 8 & & 26 & 13 & 4 & 2 yes & 85 & 38 & 30 & 9 & 6 & 2 & yes \\
\hline & 44 & 22 & 10 & 6 & 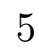 & yes & 87 & 44 & 22 & 9 & 8 & 4 yes & 87 & 44 & 21 & 10 & 10 & 2 & yes \\
\hline 87 & 44 & 20 & 12 & 10 & & yes & 87 & 44 & 18 & 17 & 4 & 4 yes & 87 & 44 & 18 & 16 & 8 & 1 & yes \\
\hline & 43 & 23 & 13 & 5 & & odd & 87 & 43 & 23 & 11 & 9 & 1 odd & 87 & 42 & 26 & 10 & 5 & & \\
\hline 8 & 42 & 20 & 20 & 4 & & yes & 87 & 41 & 28 & 8 & 6 & 4 den & 87 & 41 & 27 & 11 & 7 & 1 & odd \\
\hline 8 & 41 & 23 & 19 & 3 & & odd & 8 & 40 & 29 & 10 & 62 & 2 yes & 87 & 40 & 24 & 20 & 2 & 1 & jes \\
\hline 8 & 39 & 31 & 7 & 5 & & odd & 87 & 39 & 23 & 23 & 1 & 1 odd & 87 & 38 & 32 & 9 & 4 & & \\
\hline 8 & 36 & 34 & 10 & 5 & & yes & 87 & 36 & 34 & 8 & 8 & 1 yes & 87 & 36 & 32 & 16 & 2 & & yes \\
\hline & 35 & 35 & 9 & 7 & & & & & & & & & & & & & & & \\
\hline
\end{tabular}

Table 2: 5-splits 
THE ELECTRONIC JOURNAL OF COMBINATORICS 6 (1999), \#R25

\section{References}

[1] M. de Brandes, K.T. Phelps, V. Rödl, Coloring Steiner triple systems, SIAM J. Alg. Disc. Meth. 3 (1982), 241-249.

[2] C. J. Colbourn and J. H. Dinitz, eds. The CRC Handbook of Combinatorial Designs, CRC Press, Inc., 1996.

[3] C.J. Colbourn, A. Rosa, Triple Systems, Clarendon Press, Oxford, 1999.

[4] L. Haddad, On the chromatic numbers of Steiner triple systems, $J$. Combinat. Designs 7 (1999), 1-10.

[5] L. Milazzo, Zs. Tuza, Upper chromatic number of Steiner triple and quadruple systems, Discrete Math. 174 (1997), 247-259.

[6] L. Milazzo, Zs. Tuza, Strict colourings for classes of Steiner triple systems, Discrete Math. 182 (1998), 233-243.

[7] A. Rosa, On the chromatic number of Steiner triple systems, in: Combinat. Structures and their Applications, Proc. Conf. Calgary 1969, Gordon \& Breach 1970, pp. 369-371.

[8] A. Rosa, Steiner triple systems and their chromatic number, Acta Fac. Rer. Nat. Univer. Comen. Math. 24 (1970), 159-174.

[9] A. Rosa and C. J. Colbourn, Colorings of block designs. In Contemporary Design Theory: A Collection of Surveys, J. H. Dinitz and D. R. Stinson, eds., John Wiley \& Sons, 1992, pp. 401-430.

[10] H.J. Ryser, A combinatorial theorem with an application to Latin rectangles, Proc. Amer. Math. Soc. 2 (1951), 550-552.

[11] D.R. Stinson, Hill-climbing algorithms for the construction of combinatorial designs, Ann. Discrete Math. 26 (1985), 321-334.

[12] V.I. Voloshin, On the upper chromatic number of a hypergraph, Australas. J. Combin. 11 (1995), 25-45.

[13] D. de Werra, Equitable colorations of graphs, Rev. Francaise Automat. Informat. Rech. Oper. Ser. Rouge 3 (1971), 3-8. 